\title{
BMJ Open An observational, longitudinal study on the home environment of people with chronic obstructive pulmonary disease: the research protocol of the Home Sweet Home study
}

\author{
Nienke Nakken, ${ }^{1}$ Daisy J A Janssen, ${ }^{1,2}$ Esther H A van den Bogaart, ${ }^{1}$ \\ Jan $\mathrm{H}$ Vercoulen, ${ }^{3}$ Emiel F M Wouters, ${ }^{1,4}$ Martijn A Spruit ${ }^{1}$
}

To cite: Nakken N, Janssen DJA, van den Bogaart EHA, et al. An observational, longitudinal study on the home environment of people with chronic obstructive pulmonary disease: the research protocol of the Home Sweet Home study. BMJ Open 2014;4:e006098. doi:10.1136/bmjopen-2014006098

- Prepublication history for this paper is available online. To view these files please visit the journal online (http://dx.doi.org/10.1136/ bmjopen-2014-006098).

Received 11 July 2014 Revised 13 September 2014 Accepted 25 September 2014

CrossMark

For numbered affiliations see end of article.

Correspondence to Nienke Nakken;

nienkenakken@ciro-horn.nl

\section{ABSTRACT}

Introduction: Chronic obstructive pulmonary disease (COPD) represents an important public health challenge. Patients are confronted with limitations during activities of daily living (ADLS). Resident loved ones of patients with COPD may be uniquely positioned to witness these limitations. COPD may have an impact on not only the patients' life, but also on the lives of the resident loved ones. Furthermore, COPD exacerbation-related hospital admissions often occur in patients with COPD. However, whether and to what extent these admissions influence resident loved ones' burden and health status remains currently unknown. Therefore, the primary objectives of this study are to investigate the differences between patients with COPD and resident loved ones' perceptions of patients' health status and problematic ADLs and to study prospectively the effects of a COPD exacerbation on resident loved ones' perceptions of patients' health status and problematic ADLs.

Methods and analysis: An observational, longitudinal study will be performed in 192 patients with COPD and their 192 resident loved ones. Primary outcomes are daily functioning, ADL, disease-specific health status, generic health status and dyspnoea. These will be assessed during home visits at baseline and after 12 months. Additional home visits will be performed when a COPD exacerbation-related hospital admission occurs during the 12-month follow-up period.

Ethics and dissemination: This protocol was approved by the Medical Ethics Committee of the Catharina Hospital Eindhoven, the Netherlands (NL42721.060.12/M12-1280) and is registered in the Dutch Trial Register (NTR3941).

\section{INTRODUCTION}

Chronic obstructive pulmonary disease (COPD) is the fourth cause of death in the world $^{1}$ and represents an important public

\section{Strengths and limitations of this study}

- This study contains an individualised, clientcentred outcome measure with a semistructured interview method (the Canadian Occupational Performance Measure) to assess activities of daily living (ADLs).

- The longitudinal design of the present study will allow studying the problematic ADLs after 1-year follow-up, but also 2 weeks after a chronic obstructive pulmonary disease exacerbationrelated hospital admission.

- Recruitment of participants with an equal distributing concerning sex and disease severity will be challenging.

health challenge. ${ }^{2}$ Owing to continued exposure to COPD risk factors and aging of the population, the burden of COPD will increase in the upcoming decades. ${ }^{2}$ This, combined with the shortage in healthcare staffing $^{3}$ most probably causes a shift towards home care.

Commonly, patients with COPD suffer from multiple symptoms, like dyspnoea, coughing, sputum production, fatigue, anxiety and/or depression. ${ }^{4}$ Therefore, it is not very surprising that patients with COPD experience symptoms during activities of daily living (ADLs). ${ }^{5}{ }^{6}$ Resident loved ones may be uniquely positioned to witness the limitations that patients with COPD experience during (physical and instrumental) ADLs.

The terminology used to refer to the caregiver role is ambiguous. ${ }^{7}$ The terms 'family caregiver' or 'informal caregiver' refer to a person, often a family member or friend, who is providing care, but is not a healthcare professional. ${ }^{8}{ }^{9}$ However, informal caregivers 
themselves, in particular spouses, do not refer to the notion of being a caregiver, often describing it as an extension of their loving role and commitment to the person requiring support. ${ }^{10}{ }^{11}$ Therefore, in this study, the term resident loved one will be used. We adopt the following definition: a person living together with a patient with COPD, regardless of whether they provide care to the patient with COPD.

Caring for a loved one with advanced COPD is described as a full-time role that is akin to that of caring for people with severe disability. ${ }^{12}$ General day-to-day commitments of resident loved ones are planned around the care of the patient with COPD. ${ }^{13}$ Therefore, caregiver burden is common in family caregivers of patients with COPD. ${ }^{13}{ }^{14}$ However, caregiver burden is not associated with objective measures of the patient's need for assistance. ${ }^{13}$ In fact, caregiver burden seems more associated with the loved ones' report of need for greater help ${ }^{13}$ and symptoms of depression of the patient. ${ }^{14}$ It is known that discrepancies exist in spouses' perceptions of patients' symptoms and health status and those of the patients themselves. ${ }^{15}{ }^{16}$ How resident loved ones interact with patients with COPD probably depends on many determinants, such as their perceptions of patients' limitations in ADLs, the quality of the relationship, as well as the patients' health status and mood status. ${ }^{14} 17$ On the other hand, spouses' anxiousness is a predictor for the health status of patients with COPD. ${ }^{18}$

Family caregiving is most probably a dynamic process, in which an escalation in loved ones' anxiety, depression and psychological distress may occur as the patient's functional status declines over time or during a hospitalisation due to a COPD exacerbation. ${ }^{17} 1920$ In fact, caregivers of patients with heart failure who had fewer emergency department visits felt more positive about caregiving than other caregivers. ${ }^{21}$ Whether and to what extent COPD exacerbation-related hospital admissions influence resident loved ones' burden and health status remains currently unknown.

\section{Objectives of the study}

The primary objective of this study in patients with COPD is twofold:

1.1 To investigate the differences between patients with COPD and resident loved ones' perceptions of patients' health status and problematic ADLs.

1.2 To study prospectively the effects of a COPD exacerbation on resident loved ones' perceptions of patients' health status and problematic ADLs.

Furthermore, the following secondary objectives will be addressed:

2.1 To investigate the differences between patients with COPD and resident loved ones' perceptions of patients' mood status, care dependency and daily symptoms.

2.2 To investigate the general well-being of patients when discrepancies exist between the patients' and resident loved ones' perceptions of patients' care dependency.
2.3 To investigate if general well-being of patients and loved ones is influenced by the health or mood status of the significant other.

2.4 To study the relationship between lifestyle factors (like physical activity, smoking habit and fat free mass) in patients with COPD and their resident loved ones.

2.5 To investigate resident loved ones' burden due to patients care dependency.

2.6 To investigate resident loved ones' knowledge about COPD and the relationship with anxiety and social support.

3.1 To study prospectively the effects of a COPD exacerbation on resident loved ones' perceptions of patients' mood status, care dependency and daily symptoms.

3.2 To investigate whether and to what extent loved ones' burden and resident loved ones' health and mood status are influenced by exacerbation-related hospital admissions.

4.1 To capture differences at baseline and 12 months later between patients' and resident loved ones' perceptions of patients' health status, problematic ADLs, mood status, care dependency and daily symptoms.

The objective of this article is to show the rationale and methods of this observational, longitudinal study in patients with COPD and their resident loved ones. Furthermore, this detailed description of the research protocol of the Home Sweet Home study will serve as reference for the method section of future publications of this study. Finally, the current manuscript provides an outline of the possible strengths, weaknesses and clinical consequences.

\section{METHODS AND ANALYSIS}

\section{Study design}

An observational, longitudinal study on the home environment of people with COPD has been designed. All data will be collected during home visits at baseline and after 12 months. Additional home visits will be performed when an exacerbation-related hospital admission occurs during the 12-month follow-up period. The resident loved one will be visited extra at home $<7$ days after admission of the patient with COPD to the hospital. Finally, 2 weeks after discharge, the patient and loved one will be visited once more at home. Figure 1 gives a complete overview of the study design. Data collection will take place from July 2013 until April 2016.

\section{Eligibility criteria}

Eligible patients are those who satisfy all of the following criteria:

1. Patients with moderate to very severe COPD as main diagnosis (Global initiative for chronic Obstructive Lung Disease (GOLD) grade II, III or IV) $;^{22}$ 
2. No exacerbation of COPD (defined as 'an acute event characterised by a worsening of the patient's respiratory symptoms ie, beyond the normal day-to-day variations and leads to a change in medication' $)^{23}$ or hospitalisation $<4$ weeks preceding enrolment;

3. Provided written informed consent;

4. One resident loved one (defined as a person living together with a patient with COPD, regardless of whether they provide care to the patient with COPD) also provided written informed consent to participate.

Patients will be excluded if:

1. Patient and/or resident loved one is unable to complete the study questionnaires because of cognitive impairment (defined as Short Blessed Test score $\geq 10$ point) $; 2425$

2. Patient and/or resident loved one is unable to speak or understand Dutch.

Patients will be equally divided based on gender and GOLD grade. Furthermore, about two-third of the participating patients should be frequent exacerbators (defined as two or more COPD exacerbations, ${ }^{26}$ or one or more COPD exacerbation-related hospital admission in the year before baseline measurements). ${ }^{27}$

From eligible non-participating patients, some data like disease severity (GOLD grade), gender, and age will be collected to compare characteristics of participating and non-participating patients.

\section{Outcomes}

The following primary outcomes will be assessed at the patients' home environment: Daily functioning (Canadian Occupational Performance Measure (COPM) ${ }^{28}$ and the Instrumental Activities of Daily Living Scale (IADLS) $){ }^{29}$ disease-specific health status (COPD Assessment Test $(\mathrm{CAT})) ;{ }^{30}$ generic health status (12-Item Short Form Health Survey (SF-12), ${ }^{31}$ and the EuroQol 5-Dimensions (EQ-5D)); ${ }^{32}$ Dyspnoea (modified Medical Research Council (mMRG) dyspnoea scale).$^{33}$
In addition, the following secondary outcomes will be assessed: Symptoms of fatigue (Subjective Fatigue subscale of the Checklist Individual Strength (CIS)); ${ }^{34}$ exercise selfefficacy (self-efficacy for home walking questionnaire); symptoms of anxiety and depression (Hospital Anxiety and Depression scale (HADS) $) ;{ }^{35}$ general well-being (Assessment of Quality of Life with 8 dimensions (AQoL-8D) $) ;{ }^{36}$ mobility (Timed-Up-and-Go test) $;{ }^{37}$ daily symptoms using visual analogue scales (VAS); $;^{4}$ COPD-specific knowledge (CIROPD knowledge questionnaire); coping (Utrecht Coping List (UCL)) $;^{38}$ care dependency (Care Dependency Scale (CDS), ${ }^{39}$ and informal and professional care $<6$ months $) ;{ }^{4}$ physical activity and motivation (validated accelerometer, ${ }^{40}$ the Behavioural Regulation in Exercise Questionnaire (BREQ-2), ${ }^{41}$ and the social-individual focus); smoking status (self-developed questionnaire, and the Fagerström test for nicotine dependence) ${ }^{42}$ social support (Medical Outcome Study Social Support Survey (MOSSSS) $) ;^{43}$ quality of the relationship (Dutch relationship questionnaire (NRV)); ${ }^{44}$ cognitive functioning (Short Blessed Test $(\mathrm{SBT})){ }^{24}$ caregiver burden and positive aspects of caregiving (Family Appraisal of Caregiving Questionnaire for Palliative Care (FACQ-PC) $^{45}$ (only for resident loved ones)); clinical characteristics (fat-free mass (using body impedance assessment (BIA)), ${ }^{46}$ body weight and height, postbronchodilator spirometry, resting blood pressure, resting heart rate and resting transcutaneous oxygen saturation).

Additionally, demographics (such as age, gender, marital status and working status), medical history, (Charlson comorbidity index), ${ }^{47}$ current medication and home adaptations and aids ${ }^{4}$ will be recorded.

The resident loved one will be asked to contact the study team when an exacerbation-related hospital admission occurs during the 12-month follow-up period. Furthermore, COPD exacerbation-related hospital admissions of participants will be checked weekly in the participating hospitals by the study team.
Figure 1 Timing of the home visits. Home visits will take place in all patients and their loved ones at baseline and after 1-year follow-up. Additional home visits will be planned when a chronic obstructive pulmonary disease exacerbation-related hospital admission occurs.

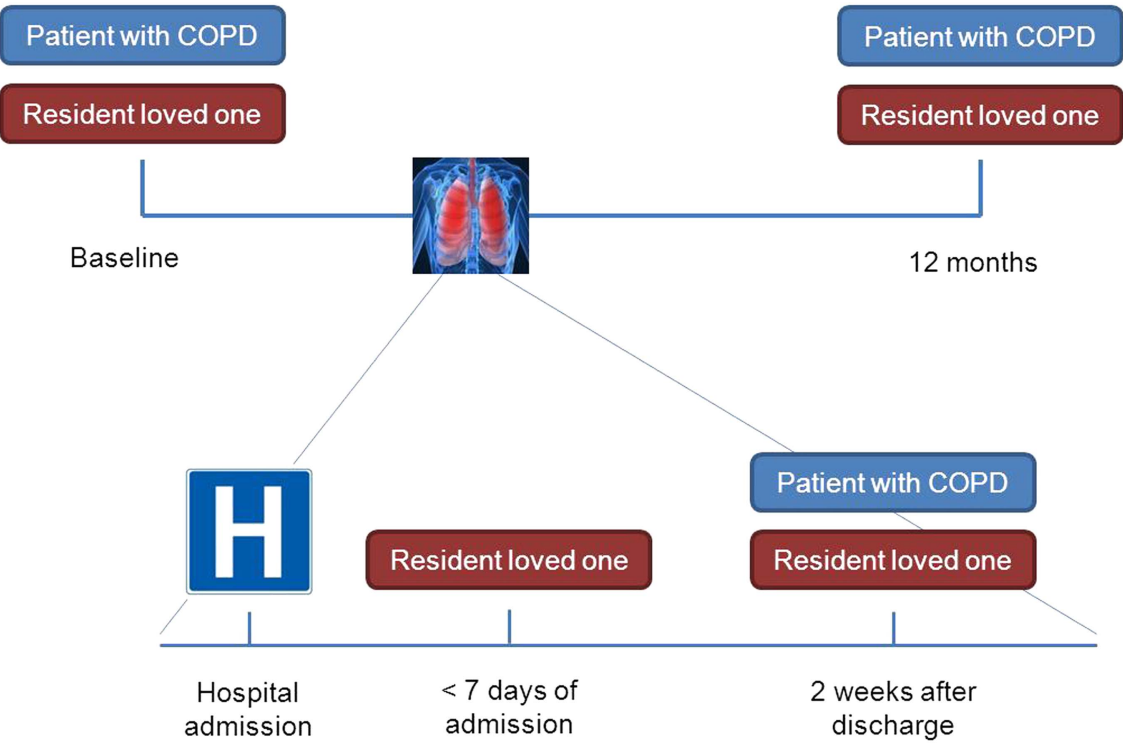


Besides questionnaires that will be administered about the patient or the resident loved one themselves, residents loved ones will be asked to complete questionnaires regarding their perception of the health status or situation of the patient. Table 1 provides an overview of the measurements per time point.

Questionnaires that were not available in Dutch have been translated into Dutch by the procedure of forwardbackward translation.

\section{Sample size}

Since no preliminary data are available concerning differences in perception in health status and ADLs, the sample size for the primary objective 1.1 is estimated using G power. A total of 171 patients and 171 resident loved ones are needed to detect an effect size of 0.25 with a significance of $5 \%$ and power of $90 \%$. Since we expect about $10 \%$ dropout, $10 \%$ additional couples will be included. Furthermore, patients will be equally divided based on gender and GOLD grade. Therefore, the study population consists of 192 patients and their resident loved ones.

For objective 1.2, 38 patients with an exacerbationrelated hospital admission during follow-up are needed to detect a medium effect size of 0.5 (significance $5 \%$ and power $85 \%$ ). In this study sample, we expect about 46 hospital admissions. ${ }^{48}$ Therefore, a sample size of 192 patients and 192 loved ones will also be sufficient to answer objective 1.2.

Table 1 Overview of measurements per home visit

Fat-free mass/height/weight

Physical activity monitor

Post-bronchodilator spirometry

Blood pressure/heart rate/saturation

Timed-up-and-go test

Cognitive test (Short Blessed Test)

Problematic activities of daily living, Canadian

Occupational Performance Measure (COPM)

Background information

Smoking history and habits

Fagerström test for nicotine dependence

COPD Assessement Test (CAT)

mMRC dyspnoea scale

Hospital Anxiety Depression Scale (HADS)

12-Item Short Form Health Survey (SF-12)

Informal and professional medical care $<6$ months

Checklist home adaptations and aids

Care Dependency Scale (CDS)

Instrumental Activities of Daily Living scale

(IADLS)

Daily symptom checklist

EuroQol 5D (EQ-5D)

Medical Outcomes Study Social Support Survey

(MOSSSS)

Charlson comorbidity index

Subjective fatigue scale (CIS)

COPD knowledge questionnaire (CIROPD)

Coping style (UCL)

Self-efficacy for home walking

Dutch relationship questionnaire (NRV)

Behavioural Regulation in Exercise Questionnaire (BREQ-2)

Social-individual focus

Assessment of Quality of Life with 8 dimensions (AQOL-8D)

Family Appraisal of Caregiving Questionnaire for Palliative Care (FACQ-PC) 


\section{Recruitment}

Patients, both with and without COPD exacerbations in the past year, will be recruited by their chest physician or a respiratory nurse during hospital admission or at the outpatient clinic in four hospitals throughout the southern-eastern part of the Netherlands. In addition, patients participating in the study 'Correlates of CAT' (NTR3416) who meet the inclusion criteria of the Home Sweet Home study and were willing to participate in further research will be asked to participate in the current study. If patients and their loved ones agree, an appointment for a first home visit will be made. Informed consent will be obtained at the start of this visit.

\section{Data management and statistical analysis}

Missing data will be minimised because all questionnaires will be completed in the presence of a researcher or research assistant. Handling of missing data will be carried out according to the guidelines of the different questionnaires. For data-analysis SPSS V.20.0 will be used.

Descriptive statistics, including means, SDs, frequencies and medians and IQRs, will be used, as appropriate. Mean scores of continuous variables will be compared between patients and their loved ones using paired sample t tests or Wilcoxon signed rank tests, depending on the variable distribution. Moreover, we will calculate intraclass correlation coefficients to study agreement between patients and loved ones in mean scores and visualise agreement using Bland and Altman plots. ${ }^{49}$ Cohen's $\kappa$ will be used to determine agreement in categorical variables. These analyses will be performed for the COPM, CAT, mMRC, SF-12, IADLS and EQ-5D. Furthermore, a mixed effect model will be used to estimate longitudinal changes. Covariates such as age and smoking status can be included as fixed effects, whereas time and exacerbation-related hospital admissions can be entered as random effects. A priori, a two-sided level of significance will be set at $\mathrm{p} \leq 0.05$.

\section{Ethics and dissemination}

This project will be conducted in accordance with the declaration of Helsinki ${ }^{50}$ and the principles of the Dutch Medical Research Involving Human Subjects Act ('WMO'). ${ }^{51}$

\section{DISCUSSION}

This study will focus on gaining knowledge about resident loved ones, including their role in the disease management and the interaction between the patient and the resident loved one in their lifestyles. This information is necessary to involve resident loved ones in the disease management of patients with COPD. The study has several strengths and limitations, which will be described below.

\section{Strengths}

The approach of this study differs from other studies on loved ones of patients with COPD, especially because of the use of unique measurements and concepts. A major strength of this study is the use of an individualised, client-centred outcome to detect problems in ADLs (COPM). ${ }^{28} \mathrm{~A}$ previous study included outcomes only based on physical activity. ${ }^{52}$ The current study detects problems in ADLs according to the patient and the loved one using a semistructured interview method. Additionally an accelerometer is used for both the patient as well as for the loved one. Moreover, these problematic ADLs are not only determined during baseline and follow-up measurements, but also 2 weeks after a COPD exacerbation-related hospital admission. The changes in these ADL problems over time and after a hospital admission are a unique point of view in this population. Furthermore, in previous studies, characteristics of the family or loved ones of the patients themselves were determined. ${ }^{17} 5354$ In this study, not only characteristics of the patients, and resident loved ones are assessed, but also the resident loved ones' perception of the patients' characteristics (such as health status, daily functioning and symptoms of anxiety and depression). Further, capabilities of loved ones (like coping styles and social support) will be investigated. In addition, these measurements are not only performed once, but will be repeated after a COPD exacerbationrelated hospital admission of the patient. Moreover, the quality of the relationship between the patient and resident loved one will be assessed. The quality of the relationship could be one of the determinants of the interaction between the resident loved one and the patient. Therefore, this study provides a complete overview of the patient, the resident loved one, and the resident loved ones' perceptions of the patient.

Other strengths of this study are related to the inclusion of the participants. Many studies focus on male patients with their female partners. ${ }^{14} 18{ }^{53}$ In this study an equal number of male and female participants will be recruited and therefore the current study will also provide knowledge concerning female patients and their male resident loved ones. Furthermore, patients will also be equally divided based on GOLD grading. Therefore, this study includes the same number of patients with moderate, severe and very severe COPD, which improves the external validity of this study.

Moreover, the present study is a longitudinal study, which makes it possible to analyse changes over time. Finally, the tests and questionnaires will all be performed at the patients' home. So participant's burden is minimised and all data will be collected in their own trusted environment.

\section{Limitations}

This study has some limitations. First, it may be possible that patients and/or loved ones are not willing to participate. Although the burden of this study is minimised, 
a possible reason for refusing participation could be the time investment. To minimise the burden of this study, all measurements are performed in the patients' homes and on a day and time of their preference. Furthermore, the home visit can be spread over 2 days. Nevertheless, it may be possible that most burdened patients and loved ones are not willing to participate in (additional) home visits. This could result in an underestimation of the burden, health status and well-being of this population. Therefore, some characteristics of the non-participating patients, like gender, age and GOLD grade, will be collected. Furthermore, it may be possible that participating resident loved ones are more aware of the situation of the patient compared to the nonparticipating loved ones. This should be taken into account in interpreting the results. Second, it might be challenging to include the same number of male and female patients with COPD. However, collaboration with four hospitals and recruitment of patients admitted to the hospital as well as patients who attend the outpatient clinic, will facilitate recruitment. Third, some loved ones of admitted patients may be unwilling to participate in additional home visits shortly after the patients' admission to the hospital. This could lead to missing data or even drop-outs. However, the time investment and impact of this visit is minimal. Fourth, the follow-up period is limited to 1 year. This follow-up period could be too short to draw conclusions about long-term changes in patients' health status and problems during ADLs and the perception of the resident loved ones about these changes. Finally, it may be possible that participation in this study works as a stimulus for patients to talk about COPD with their loved ones. This could result in more understanding and agreement during the measurements after 12 months. However, if participants communicate more about the disease and the problems during daily activities, the expectation is that this will be performed shortly after the first home visit.

\section{Clinical consequences}

The present study will gain more knowledge about the resident loved ones of patients with COPD and about their perceptions of the patients' health status and problematic ADLs. With this information, a more systemic approach in the treatment of patients with COPD could be developed. Healthcare providers should not only focus on the patient, but should see the patient, his/her loved ones and their interaction as a whole. Only by gaining more information about the loved ones themselves, the quality of the relationship, and their perception of the patients' health status and problematic ADLs, loved ones could be involved in the patient's treatment. With the knowledge gained in this study, we will learn about how to carry out self-management plans in patients and loved ones, so loved ones are able to facilitate and encourage the self-management. Furthermore, this study investigates the impact of a hospitalisation due to an acute COPD exacerbation on the resident loved ones. With this information it may be possible to determine whether and how professional caregivers should give more attention to the resident loved ones during an exacerbation related hospitalisation.

\section{CONCLUSION}

In conclusion, COPD may have an impact on not only the patients' life, but also on the lives of the resident loved ones. As a shift towards home care is anticipated, the Home Sweet Home study is necessary to give more insight in the home situation of patients with COPD.

\section{Author affiliations}

${ }^{1}$ Department of Research and Education, CIR0+, Centre of Expertise for Chronic Organ Failure, Horn, The Netherlands

${ }^{2}$ Centre of expertise for palliative care, Maastricht University Medical Centre+ (MUMC+), Maastricht, The Netherlands

${ }^{3}$ Department of Medical Psychology and Department of Pulmonary Diseases, Radboud University Nijmegen Medical Centre, Nijmegen, The Netherlands ${ }^{4}$ Department of Respiratory Medicine, Maastricht University Medical Centre+ (MUMC+), Maastricht, The Netherlands

Contributors NN, DJAJ, EFMW and MAS designed and established the study. All authors contributed to the writing of this manuscript, read and approved the final version of the manuscript.

Funding The Home Sweet Home study was supported by Grant 3.4.12.024 of Lung Foundation Netherlands, Leusden, The Netherlands and has received a research grant from Boehringer-Ingelheim Netherlands.

\section{Competing interests None.}

\section{Patient consent Obtained.}

Ethics approval Ethics approval has been obtained from the Medical Ethical Committee of the Catharina hospital Eindhoven, The Netherlands (NL42721. 060.12/M12-1280) and is registered in the Dutch trial register (NTR3941).

Provenance and peer review Not commissioned; externally peer reviewed.

Open Access This is an Open Access article distributed in accordance with the Creative Commons Attribution Non Commercial (CC BY-NC 4.0) license, which permits others to distribute, remix, adapt, build upon this work noncommercially, and license their derivative works on different terms, provided the original work is properly cited and the use is non-commercial. See: http:// creativecommons.org/licenses/by-nc/4.0/

\section{REFERENCES}

1. World Health Organization. The 10 leading causes of death in the world, 2000 and 2011. Secondary World Health Organization. The 10 leading causes of death in the world, 2000 and 2011 July 2013 2013. http://www.who.int/mediacentre/factsheets/fs310/en/index.html

2. Vestbo J, Hurd SS, Agusti AG, et al. Global strategy for the diagnosis, management, and prevention of chronic obstructive pulmonary disease: GOLD executive summary. Am J Respir Crit Care Med 2013;187:347-65.

3. Heinen MM, van Achterberg T, Schwendimann R, et al. Nurses' intention to leave their profession: a cross sectional observational study in 10 European countries. Int J Nurs Stud 2013;50:174-84.

4. Janssen DJ, Spruit MA, Uszko-Lencer NH, et al. Symptoms, comorbidities, and health care in advanced chronic obstructive pulmonary disease or chronic heart failure. $J$ Palliat Med 2011;14:735-43.

5. Vaes AW, Wouters EF, Franssen FM, et al. Task-related oxygen uptake during domestic activities of daily life in patients with COPD and healthy elderly subjects. Chest 2011;140:970-9.

6. Annegarn J, Meijer K, Passos VL, et al. Problematic activities of daily life are weakly associated with clinical characteristics in COPD. J Am Med Dir Assoc 2012;13:284-90.

7. Spence A, Hasson F, Waldron M, et al. Active carers: living with chronic obstructive pulmonary disease. Int J Palliat Nurs 2008;14:368-72. 
8. Houts PS, Nezu AM, Nezu CM, et al. The prepared family caregiver: a problem-solving approach to family caregiver education. Patient Educ Couns 1996;27:63-73.

9. Candy B, Jones L, Drake R, et al. Interventions for supporting informal caregivers of patients in the terminal phase of a disease. Cochrane Database Syst Rev 2011;(6):CD007617.

10. Bergs D. "The Hidden Client"-women caring for husbands with COPD: their experience of quality of life. J Clin Nurs 2002;11:613-21.

11. Seamark DA, Blake SD, Seamark CJ, et al. Living with severe chronic obstructive pulmonary disease (COPD): perceptions of patients and their carers. An interpretative phenomenological analysis. Palliat Med 2004;18:619-25.

12. Hynes G, Stokes A, McCarron M. Informal care-giving in advanced chronic obstructive pulmonary disease: lay knowledge and experience. J Clin Nurs 2012;21:1068-77.

13. Garlo K, O'Leary JR, Van Ness PH, et al. Burden in caregivers of older adults with advanced illness. J Am Geriatr Soc 2010;58:2315-22.

14. Janssen DJ, Spruit MA, Wouters EF, et al. Family caregiving in advanced chronic organ failure. J Am Med Dir Assoc 2012;13:394-9.

15. Low G, Gutman G. Couples' ratings of chronic obstructive pulmonary disease patients' quality of life. Clin Nurs Res 2003;12:28-48.

16. Janssen DJ, Spruit MA, Wouters EF, et al. Symptom distress in advanced chronic organ failure: disagreement among patients and family caregivers. J Palliat Med 2012;15:447-56.

17. Jacome C, Figueiredo D, Gabriel R, et al. Predicting anxiety and depression among family carers of people with chronic obstructive pulmonary disease. Int Psychogeriatr 2014;26:1191-9.

18. Kuhl K, Schurmann W, Rief W. Mental disorders and quality of life in COPD patients and their spouses. Int $J$ Chron Obstruct Pulmon Dis 2008;3:727-36.

19. Fitzsimons D, Mullan D, Wilson JS, et al. The challenge of patients' unmet palliative care needs in the final stages of chronic illness. Palliat Med 2007;21:313-22.

20. Figueiredo D, Gabriel R, Jacome C, et al. Caring for relatives with chronic obstructive pulmonary disease: how does the disease severity impact on family carers? Aging Ment Health 2014;18:385-93

21. Hwang B, Fleischmann KE, Howie-Esquivel J, et al. Caregiving for patients with heart failure: impact on patients' families. Am J Crit Care 2011;20:431-41; quiz 42

22. Vestbo J, Hurd SS, Agusti AG, et al. Global Strategy for the Diagnosis, Management and Prevention of Chronic Obstructive Pulmonary Disease, GOLD Executive Summary. Am J Respir Crit Care Med 2013;187:347-65

23. Rodriguez-Roisin R. Toward a consensus definition for COPD exacerbations. Chest 2000;117(5 Suppl 2):398S-401S.

24. Carpenter CR, Bassett ER, Fischer GM, et al. Four sensitive screening tools to detect cognitive dysfunction in geriatric emergency department patients: brief Alzheimer's Screen, Short Blessed Test, Ottawa 3DY, and the caregiver-completed AD8. Acad Emerg Med 2011;18:374-84

25. Morris JC, Heyman A, Mohs RC, et al. The Consortium to Establish a Registry for Alzheimer's Disease (CERAD). Part I. Clinical and neuropsychological assessment of Alzheimer's disease. Neurology 1989;39:1159-65.

26. Hurst JR, Vestbo J, Anzueto A, et al. Susceptibility to exacerbation in chronic obstructive pulmonary disease. N Engl J Med 2010;363:1128-38.

27. Global Strategy for the Diagnosis MaPoC. Global Initiative for Chronic Obstructive Lung Disease (GOLD) 2014. Secondary Global Initiative for Chronic Obstructive Lung Disease (GOLD) 20142014. http://www.goldcopd.org/

28. Dedding C, Cardol M, Eyssen IC, et al. Validity of the Canadian Occupational Performance Measure: a client-centred outcome measurement. Clin Rehabil 2004;18:660-7.

29. Lawton MP, Brody EM. Assessment of older people: self-maintaining and instrumental activities of daily living. Gerontologist 1969;9:179-86.

30. Jones PW, Harding G, Berry P, et al. Development and first validation of the COPD Assessment Test. Eur Respir $J$ 2009;34:648-54.
31. Resnick B, Nahm ES. Reliability and validity testing of the revised 12-item Short-Form Health Survey in older adults. J Nurs Meas 2001;9:151-61.

32. Pickard AS, Wilke $C$, Jung $E$, et al. Use of a preference-based measure of health (EQ-5D) in COPD and asthma. Respir Med 2008;102:519-36

33. Bestall JC, Paul EA, Garrod R, et al. Usefulness of the Medical Research Council (MRC) dyspnoea scale as a measure of disability in patients with chronic obstructive pulmonary disease. Thorax 1999;54:581-6.

34. Vercoulen JH, Swanink CM, Fennis JF, et al. Dimensional assessment of chronic fatigue syndrome. J Psychosom Res 1994;38:383-92.

35. Zigmond AS, Snaith RP. The hospital anxiety and depression scale. Acta Psychiatr Scand 1983;67:361-70.

36. Richardson J, Lezzi A. Psychometric validity and the AQoL-8D multi attribute utility instrument. Centre for Health Economics. Melbourne, Australia: Monash University, Business and Economics, 2011.

37. Podsiadlo D, Richardson S. The timed "Up \& Go": a test of basic functional mobility for frail elderly persons. J Am Geriatr Soc 1991;39:142-8.

38. Scharloo M, Kaptein AA, Weinman J, et al. Illness perceptions, coping and functioning in patients with rheumatoid arthritis, chronic obstructive pulmonary disease and psoriasis. J Psychosom Res 1998;44:573-85.

39. Dijkstra A, Tiesinga LJ, Goossen WT, et al. Further psychometric testing of the Dutch Care Dependency Scale on two different patient groups. Int J Nurs Pract 2002;8:305-14.

40. Annegarn J, Spruit MA, Uszko-Lencer $\mathrm{NH}$, et al. Objective physical activity assessment in patients with chronic organ failure: a validation study of a new single-unit activity monitor. Arch Phys Med Rehabil 2011;92:1852-57. e1.

41. Markland $D$, Tobin V. A modification to the behavioural regulation in exercise questionnaire to include an assessment of amotivation. J Sport Exerc Psychol 2004;26:6.

42. Heatherton TF, Kozlowski LT, Frecker RC, et al. The Fagerstrom Test for Nicotine Dependence: a revision of the Fagerstrom Tolerance Questionnaire. Br J Addict 1991;86:1119-27.

43. Sherbourne CD, Stewart AL. The MOS social support survey. Soc Sci Med 1991;32:705-14.

44. Barelds DPH, Luteijn F, Arrindell WA. Nederlandse Relatievragenlijst (NRV). In: PAal BV, ed. Amsterdam: Handleiding, 2003.

45. Cooper B, Kinsella GJ, Picton C. Development and initial validation of a family appraisal of caregiving questionnaire for palliative care. Psychooncology 2006;15:613-22.

46. Rutten EP, Spruit MA, Wouters EF. Critical view on diagnosing muscle wasting by single-frequency bio-electrical impedance in COPD. Respir Med 2010;104:91-8.

47. Charlson ME, Pompei P, Ales KL, et al. A new method of classifying prognostic comorbidity in longitudinal studies: development and validation. J Chronic Dis 1987;40:373-83.

48. Miravitlles M, Mayordomo C, Artes M, et al. Treatment of chronic obstructive pulmonary disease and its exacerbations in general practice. EOLO Group. Estudio Observacional de la Limitacion Obstructiva al Flujo aEreo. Respir Med 1999;93:173-9.

49. Bland JM, Altman DG. Statistical methods for assessing agreement between two methods of clinical measurement. Lancet 1986;1:307-10.

50. World Medical Association Declaration of Helsinki. Recommendations guiding physicians in biomedical research involving human subjects. JAMA 1997;277:925-6.

51. Wet van 26 februari 1998, houdende regelen inzake medisch-wetenschappelijk onderzoek met mensen (Wet medisch-wetenschappelijk onderzoek met mensen). 28-02-1998. http://wetten.overheid.nl

52. Barriga S, Rodrigues F, Barbara C. Factors that influence physical activity in the daily life of male patients with chronic obstructive pulmonary disease. Rev Port Pneumol 2014;20:131-7.

53. Figueiredo D, Gabriel R, Jacome C, et al. Caring for people with early and advanced chronic obstructive pulmonary disease: how do family carers cope? J Clin Nurs 2014;23:211-20.

54. Kuprys-Lipinska I, Kuna P. Impact of chronic obstructive pulmonary disease (COPD) on patient's life and his family. Pneumonol Alergol Pol 2014;82:82-95. 